\title{
A review on treatment of Alzheimer's disease
}

\section{Suman Pratihar*, Shomik Nag and Jayanta Kumar Kundu}

Molecular Biology Unit, Department of Zoology, Vidyasagar University, Midnapore (W.B), INDIA

"Corresponding author. E-mail: pratihar-_vu@yahoo.co.in

Abstract: Alzheimer's disease is a brain disorder named after German physician Alois Alzheimer, who first described it in 1906. The Alzheimer's disease is now becoming curable by identifying the actual cause of the disease with the proper diagnosis of the disease of the patient. The disease can now be cured by drug treatment, by hormonal treatment and by using herbal products such as medicines. The drugs are based on antagonizing action of the components against the causative agents (â Amyloid plaque, Apolipoprotein E, Tau protein) of the disease. The common drugs based on their action are N-methyl-D-aspartate (NMDA) receptor antagonists, Cholinesterase inhibitor, agents for increasing blood supply and neurotransmitter in the brain, lipofuchsin and â Amyloid plaque inhibitor, free radical scavenger, agent for metal chelator and agent for increasing macrophage activity in the brain.

Keywords: Alzheimer's disease, Memantine, Hydergine, Rivastigmine tartrate, Turmeric

\section{INTRODUCTION}

Alzheimer's disease (AD) also called Alzheimer disease, Senile Dementia of the Alzheimer Type (SDAT) or simply Alzheimer's is the most common form of dementia where dementia (from Latin de- "apart, away" + mens "mind") is the progressive decline in cognitive function due to damage or disease in the body beyond what might be expected from normal aging. Generally it is diagnosed in people over 65 years of age, although the less-prevalent early-onset Alzheimer's can occur much earlier. An estimated 26.6 million people worldwide had Alzheimer's in 2006; this number may quadruple by 2050 (Brookmeyer et al., 2007). This degenerative and terminal disease was first described by German psychiatrist Alois Alzheimer in 1906 by observing the patient Auguste D.

Modern research indicates that the disease is associated with plaques and tangles in the brain (Tiraboschi et al., 2004).Brain atrophy reveal the damage caused by Alzheimer's disease. Certain regions of the brainsincluding the hippocampus and cortex - lose functions and the normally convoluted surface of the brain ultimately wastes away. Various studies have found that groups of people exposed to high levels of aluminum do not have an increased risk. Moreover, aluminum in cooking utensils does not get into food and the aluminum that does occur naturally in some foods, such as potatoes, is not absorbed well by the body. On the whole, scientists can say only that it is still uncertain and rather unlikely that exposure to aluminum plays a role in Alzheimer's disease. On the other hand, a recent study suggests that too much zinc might be the problem. In an experiment, zinc caused soluble beta amyloid from cerebrospinal fluid to form clumps similar to the plaques of Alzheimer's disease.

The plaques, often known as â amyloid plaques are focal, spherical, collections of dilated, tortuous, silver staining, neuritic process (dystrophic neurites) often around a central amyloid core, which may be surrounded by clear halo. Plaques can be found in the hippocampus and amygdale as well as in the neocortex. The amyloid core which can be stained by Congo red, contains several abnormal proteins (like as Amyloid Precursor protein, Apolipoprotein E and Tau Protein). However, Neurofibrillary tangles are bundles of filaments in the cytoplasm of the neurons that displace or encircle the nucleus They are commonly found in cortical neurons, especially in the entorhinal cortex, as well as in other in other sites such as in other sites such as pyramidal cells, as well as in pyramidal cells of the hippocampus, the amygdala, the basal forebrain and the raphe nuclei. In pyramidal neurons, they often have an elongated "flame" shape; in rounder cells, the basket wave of fibers around the nucleus take on a rounded contour. They are visible in silver staining.

\section{CAUSES OF ALZHEIMER'S DISEASE}

According to Amyloid hypothesis, following factors are responsible for Alzheimer's disease:

A. Role of amyloid precursor protein in alzheimer's disease: Amyloid Precursor Protein (APP) is a 695-770 amino acid long transmembrane protein. The gene for this protein is situated on chromosome 21.It has cleavage site for three enzymes which are- â secretase, á secretase and ã- secretase. Normally âAPP is cut twice, first by â 
secretase to C99-âAPP, then by ã secretase to either 40 â amyloid protein (normally $90 \%$ produced) or 42 â amyloid protein (normally $10 \%$ produced). Mutation in âAPP gene found and believed to produce a form of the protein with shifted amino acids which guide â secretase followed by ã secretase to cut the protein preferentially into 42 â amyloid protein which can aggregates and form fibrils. As APP is encoded from chromosome 21, thus peoples with Down's syndrome have an increased risk of developing familial Alzheimer's disease. (Lott and Head, 2005).

B. Role of apo lipoprotein $E$ in alzheimer's disease: Apo lipoprotein $\mathrm{E}$ is coded by the gene APOE on chromosome 19 and it comes in three forms -å2, å3, å4. It plays many important roles in body like as- transports cholesterol in body, helps in cellular repair and regeneration. Amyloid proteins are transported into the cell by membrane bound protein transporters. In AD there appears to be an interface with transport of 42 âamyloid protein by APOE (Apolipoprotein å4 form). Toxic â amyloid fragments builds up outside the cell. Sometimes the å4 form of Apolipoprotein (APOE) is selectively removed from extracellular space instead of the âamyloid-leaving the latter to cause mischief.

C. Role of tau protein in alzheimer's disease: Tau proteins interact with tubulin to stabilize microtubules and promote tubulin assembly into microtubules. Tau controlls microtubule stability by phosphorylation which is regulated by a host of kinases. Hyperphosphorylation of the tau protein (tau inclusions), can result in the selfassembly of tangles of paired helical filaments and straight filaments, forming "Neuro fibrillary tangles" (Hernández and Avila, 2007).

\section{PROTEOMICS OF ALZHEIMER'S DISEASE}

Dr. van Leeuwan by discovering a new type of mutation suggested that due to molecular reading errors, transcripts (mRNA) may arise in which two nucleotide are missing. If these transcripts are translated in the +1 reading frame and the proteins do not decompose, they may accumulate. Indeed this appeared to be the case: in

Table 1. Genetics of alzheimer's disease.

\begin{tabular}{|c|c|c|c|}
\hline Chromosome & Gene & Mutations/Alleles & Consequences \\
\hline 21 & Amyloid Precursor Protein & $\begin{array}{l}\text { 1) Single missense mutations } \\
\text { 2) Double missense mutations } \\
\text { 3) Trisomy 21(gene dosage effect) }\end{array}$ & $\begin{array}{l}\text { 1)Early-onset } A D \\
\text { 2)Increased } A \beta \text { production }\end{array}$ \\
\hline 14 & Presenilin (PS1) & $\begin{array}{l}\text { 1) Missense mutations } \\
\text { 2) Splice site mutations }\end{array}$ & $\begin{array}{l}\text { 1) Early-onset } A D \\
\text { 2) Increased } A \beta \text { production }\end{array}$ \\
\hline 1 & Presenilin(PS2) & Missense mutations & $\begin{array}{l}\text { 1) Early-onset } F A D \\
\text { 2) Increased } A \beta \text { production }\end{array}$ \\
\hline 19 & Apolipoprotein E(Apo E) & Allele $\varepsilon 4$ & $\begin{array}{l}\text { 1) Increased risk of development of } A D \\
\text { 2) Decreased age at onset of } A D\end{array}$ \\
\hline
\end{tabular}

the characteristics of the disease (among other things neurofibrillary tangles and neuritic plaques) of all Alzheimer patients two of these ' +1 proteins', i.e. ubiquitin $\mathrm{B}+1$ and amyloid precursor protein $+1(\mathrm{UBB}+1$ and $A P P+1$ ) have been found. Meanwhile the researchers have demonstrated that UBB +1 inhibit the action of the proteasome. The proteasome is involved in the enzymatic decomposition of many (aberrating) proteins. A not well functioning ubiquitin-proteasome system may be one of the causes that eventually nerve cells become less active and even die. $\mathrm{UBB}+1$ is an important chain in the neurotoxicity of the beta-amyloid protein (present in plaques) (Leeuwan, 2001).

\section{SYMPTOMS OF ALZHEIMER'S DISEASE}

Following are the different stages of Alzheimer's disease with symptoms:

Predementa: The most noticeable deficit is memory loss, which shows up as difficulty in remembering recently learned facts and inability to acquire new information. (Arnáiz and Almkvist., 2003) Subtle problems with the executive functions of attentiveness, planning, flexibility, and abstract thinking, or impairments in semantic memory, can also be symptomatic of the early stages of AD. Apathy can be observed at this stage.

Early dementia: In this stage difficulties with language, executive functions, perception (agnosia), or execution of movements (apraxia) are more prominent than memory problems (Forstl and Kurz, 1999). Language problems are mainly characterized by a shrinking vocabulary and decreased word fluency. While performing fine motor tasks such as writing, drawing or dressing, certain movement coordination and planning difficulties (apraxia) may be present, making sufferers appear clumsy (Benke, 1993).

Moderate dementia: During this phase complex motor sequences become less coordinated, reducing the ability to perform most normal daily living activities, memory problems worsen, and the person may fail to recognize close relatives. Common neuropsychiatric manifestations are wandering, sun downing, irritability and labile affect, leading to crying, outbursts of unpremeditated 
aggression, or resistance to care giving.

Adavanced dementia: In this stage, patients will ultimately not be able to perform even the simplest tasks without assistance. Muscle mass and mobility deteriorate to the point where they are bedridden, and they lose the ability to feed themselves. Finally comes death, usually caused directly pressure ulcers or pneumonia etc.

\section{DIAGNOSIS OF ALZHEIMER'S DISEASE}

Detecing alzheimer's by eye test: The amyloid beta proteins which are a hallmark of Alzheimer's are also found in the lens and fluid of the eye. These proteins produce an unusual type of cataract in a different part of the eye. Scientists can detect these proteins by injecting a lightsensitive dye, then shining a laser onto the specific part of the lens where the cataracts tend to form. The molecules in the dye bind to the protein molecules, if they are present, and the light will cause the resulting molecules to glow. This technique is called quasi-elastic light scattering.

Detecting alzheimer's by positron emission tomography scan: In this technique patients are injected with a radioactive dye called Pittsburgh Compound-B-or PIB. Once it reaches the brain, PIB attaches to plaque. Then, a PET scan reveals areas with the most plaque build-up. The pattern of PIB retention in the brain suggests that amyloid plaques formed by Alzheimer's appearing first in the frontal cortex areas, then progressing to the parietal and temporal cortex before ravaging the occipital and sensory-motor cortex. (Kemppainen et al., 2008)

Other techniques like-EEG Test, MRI Scan etc. are also used for diagnosis of the disease.

\section{TREATMENTSOFALZHEIMER'S DISEASE}

There are some drugs which are now proven to be effective for the treatment of Alzheimer's disease. They are like as Memantine, Hydergine, Exelon, Ginkgo biloba extracts (Oyama et al., 1996) etc. Beside these, different natural products such as Turmeric (Fiala et al., 2007) and hormonal treatments are also useful.

A. Memantine:Memantine is the first representative of a moderate affinity NMDA receptor antagonist. NMDA receptor is a type of ionotropic receptor which is present in brain and plays an important role in mediating plastic changes in brain .It has following features -

I. It is highly permeable to $\mathrm{Ca}^{2+}$ ions.

II. It has voltage dependant blockage by $\mathrm{Mg}^{2+}$ ions.

III. It shows slow gating kinetics.

Mode of action: Under normal conditions, learning is based on detection of sufficiently strong signal over baseline activity i.e. sufficient signal-to-noise ratio. Signal-to-noise ratio hypothesis assumes that in Alzheimer's disease, due to overactive glutamatergic system, $\mathrm{Mg}^{2+}$ is not effective enough to play its 'filtering' function. In turn, synaptic noise rises, impairing detection

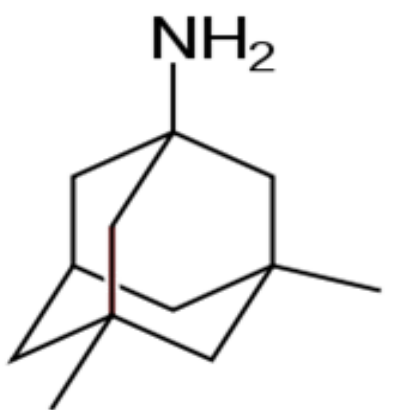

Fig. 1. Chemical structure of Memantine.

of the relevant signal such as in learning. Memantine is able to serve as a filter blocking 'synaptic noise' and thereby allowing detection of the relevant signal i.e. synaptic plasticity is restored. (Danysz and Parsons, 2003) Side effects: The side effects of using Memantine as drug for Alzheimer's disease are as follows:

I.Common symptoms: Dizziness, constipation, hypertension, headache, somnolence etc.

II.Uncommon symptoms: Gait, Vomiting, thromboembolism, fatigue, hallucinations, confusion.

B. Ergoloid mesylates :Ergoloid mesylates is a mixture of the methanesulfonate salts of three dihydrogenated ergot alkaloids (dihydroergocristine, dihydroergocornine, and alpha- and beta-dihydroergocryptine). It is now present in market in different brand names like-Hydergine, Hydergina, Gerimal, Niloric, Redizork, Alkergot, Cicanol, Redergin etc. The most important among them is Hydragine.

\section{Mechanism of action of Hydragine:}

I. Hydergine increase blood supply to the brain.

II. Hydergine slows the deposit of the age pigment lipofuscin in the brain.

III. It Increase oxygen delivered to the brain and protects the brain from insufficient oxygen supply and also prevents free radical damage to brain cells (Spiegel, 1983). IV. Hydergine has also been shown to increase the level of neurotransmitters in the brain,

V. Hydergine enhances the metabolism of brain cells. There is also evidence that hydergine stimulates the growth of dendrite nerve fibers which is normally be expected to decline with aging.

VI. In experiments it has been found that in young adult rats, the energy required at synaptic regions are provided by a large number of small, highly efficient mitochondria, whereas in old rats, energy is produced by a smaller number of larger, less efficient mitochondria. But, after treatment with Hydergine, it can be seen that the total mitochondrial volume of old rats was nearly the same as the young rats. Furthermore, the mitochondrial size was altered to a more youthful direction

VII. Hydergine has also shown itself to be a mild vasodilator (it enhances brain blood flow) and improves the uptake of the brain energy molecule - glucose. 
Hydergine also reduces the accumulation of age-related toxin, lipofuscin.

Side effects: Hydergine has proven itself to be non-toxic and relatively safe although its potential side effects include mild nausea, gastric disturbances and bradycardia and stomach upset.

\section{Exelon (Rivastigmine tartrate): Exelon (rivastigmine} tartrate) is a cholinesterase inhibitor. Exelon is specifically indicated for the treatment of mild to moderate dementia of the Alzheimer's type and for the treatment of mild to moderate dementia associated with Alzheimer's disease.

Mode of action of exelon: Based on their structure and mode of enzyme inhibition, ChEIs can be grouped into three classes: tertiary and quaternary amines, carbamates, and organophosphates. Acetyl cholinesterase (AChE), the enzyme that breaks down $\mathrm{ACh}$, is well characterized. It has two structural binding sites for $\mathrm{ACh}$, an anionic site and an esteratic site, in two- and three-dimensional configurations. The ChEIs are classified according to their duration of enzyme inhibition as short-acting, intermediate-acting, or long-acting inhibitors. In contrast to Exelon, Tacrine and donepezil are short-acting ChEI that bind to $\mathrm{AChE}$ by hydrogen bonding and are hydrolyzed within minutes by the body's water. Both rivastigmine and tacrine bind to $\mathrm{AChE}$, however, rivastigmine fits into the enzyme's active site in a similar fashion to ACh, which results in a "flattening" of the carbamate moiety over the esteratic site, producing the prolonged inhibition of AChE.

Like tacrine and donepezil, rivastigmine is hydrolyzed, but unlike these two agents, it leaves the esteratic site carbamylated and the phenolic derivative is excreted rapidly from the body. Sequestration of $\mathrm{AChE}$ in the carbamylated form precludes further enzyme hydrolysis of ACh. On administration of a single dose, the carbamate moiety can inhibit enzyme activity up to 10 hours. Due to this longer duration of action, rivastigmine is classified as an intermediate-acting (pseudo-irreversible) agent.

Side effects :Adverse events associated with the use of Exelon patch may include nausea, vomiting, diarrhea, depression, headache, anxiety, anorexia, weight loss etc. D. Ginkgo biloba:Ginkgo biloba is a type of tree that has existed for over 200 million years. The extract of dried leaves of this tree has been shown to have protective effects against mitochondrial damage and oxidative stress. There are different variants of Ginkgo biloba extracts available on the market today. Among them are EGb 761, LI 1379, and Chinese Ginkgo extract ZGE. Ginkgo biloba is commonly used in the treatment of early-stage Alzheimer's disease, vascular dementia, peripheral claudication, and tinnitus of vascular origin. Multiple trials investigating the efficacy of ginkgo for treating cerebrovascular disease and dementia have been performed, and systematic reviews suggest the herb can improve the symptoms of dementia (Oyama et al., 1996). Mechanism of action of Ginkgo biloba: The mechanism of action of ginkgo is believed to be produced by its functions as a neuroprotective agent, an antioxidant, a free-radical scavenger, a membrane stabilizer, and an inhibitor of platelet-activating factor via the terpene ginkgolide B. The different components of Ginkgo biloba like as Flavonoids contributes to its antioxidant property \& Bilobalides helps to protects nerve cells and regenerates nerve cells. Other pharmacologic effects include the following: endothelium relaxation mediated by inhibition of 3', 5'-cyclic GMP (guanosine monophosphate) phosphodiesterase, inhibition of agerelated loss of muscarinergic cholinoceptors and adrenoceptors; and stimulation of choline uptake in the hippocampus. Ginkgo extract also has been shown to inhibit beta-amyloid deposition. Some scientist hypothesized that Ginkgo biloba treatment would have beneficial effects on cells exposed to the free radical hydrogen peroxide (Oyama et al., 1996).

E. Turmeric:Turmeric is best known as one of the ingredients used to make curry, it also gives ballpark mustard its bright yellow color. The turmeric plant (Curcuma longa or Curcuma domestica) is a member of the ginger family. It has aromatic, somewhat fleshy rhizomes that, when dried, yield a bright yellow powder commonly used as a spice or coloring agent - sometimes both, as in certain yellow mustards.

In herbal medicine, turmeric (in the form of an extract of Curcuma longa) is known for its action as: Antihapatotoxic, Anti-hyperlipidemic, Anti-inflammatory, Antitumoral, Antimicrobial, Antifertile and mainly for its antioxidant property.

Role of turmeric preventing alzheimer's disease:

I.Curcumin is a polyphenolic diketone from turmeric. Because of its anti-oxidant and anti-inflammatory effects, it can reduce levels of amyloid and oxidized proteins and can prevent cognitive deficits. It can also reduce amyloid aggregation by metal chelation. Metals can induce Aâ aggregation and toxicity, and are concentrated in $\mathrm{AD}$ brain. Using spectrophotometry, curcumin's affinity for copper, zinc, and iron ions has been quantified. $\mathrm{Zn}^{2+}$ showed little binding, but each $\mathrm{Cu}^{2+}$ or $\mathrm{Fe}^{2+}$ ion appeared to bind at least two curcumin molecules. Since curcumin more readily binds the redox-active metals iron and copper than redox-inactive zinc, curcumin might exert a net protective effect against Aâ toxicity or might suppress inflammatory damage by preventing metal induction of NF-êB.

II. Curcumin may interrupt the production of IL-2, a protein that can play a key role in the destruction of myelin, the sheath that serves to protect most nerves in the body. III. Different studies have suggested that curcumin turns on a gene that code for the production of antioxidant 
proteins. A study showed curcumin's role in the induction of the the heme oxygenase pathway, that produces the potent antioxidant bilirubin, which protects the brain against oxidative injury. Such oxidation is thought to be a major factor in Alzheimer's disease. (Calabrese et al., 2003) Another study confirmed that, curcumin strongly induces expression of hemeoxygenase-1 (HO-1) gene in astrocytes from the hippocampal region of the brain.

IV. Using blood samples from Alzheimer's patients, Fiala et al., 2007 have shown that bisdemethoxycurcumin boosts macrophage activity to normal levels, helping to clear amyloid beta. They also observed that bisdemethoxycurcumin was more effective in promoting the clearance of amyloid beta in some patients' blood than others, hinting at a genetic element. Further study revealed the genes involved are MGAT III and Toll-like receptors, which are also responsible for a number of other key immune functions. Bisdemethoxycurcumin enhances the transcription of these genes, correcting the immune defects seen in Alzheimer's patients.

\section{ROLEOFVITAMINSINPREVENTINGALZHEIMER'S} DISEASE

Niacin: A study examined whether dietary vitamin B3 (niacin) could prevent cognitive decline and Alzheimer's disease, suggests that higher intake of niacin did appear to be associated with a slower annual rate of cognitive decline, leading the researchers to conclude that dietary niacin may actually protect against Alzheimer's disease. Vitamin E: Scientists has demonstrated that in the animal form of the amyloid beta peptide, the antioxidant vitamin E slowed the destruction of brain cells as it appears to in the human form, but the patients may have complain of nausea, intestinal cramping, fatigue or blurred vision(Kappus and Diplock, 1992).

\section{ROLE OF HORMONES IN PREVENTING ALZHEIMER'SDISEASE}

Estrogen as alzheimer's preventer: Animal studies have shown that administration of estrogen to estrogendeficient laboratory animals restores the number of neural synapses, causes beta-amyloid to be more soluble and has an antioxidant effect. A recent descriptive study showed a lower relative risk of Alzheimer's disease and a later onset of dementia in those who had taken estrogen, compared with those who had not. The apparent benefit was increased if the length of treatment was more than one year (Goodman et al., 1996).

New cancer drug may help alzhemiers disease : A new study shows that the experimental drug BRYOSTATIN that is proved to be useful in cancer prevention stimulates proteins needed to form long-term memories, an ability lost in the early stages of Alzheimer's disease. Researchers found treating sea slugs (Hermissenda mollusks) with the drug-BRYOSTATIN, days before a new learning activity significantly increased their long-term memory of the skill. Instead of remembering the skill for a few minutes as usual, they remembered it for weeks.

Drug for renal disorder cures alzheimers's disease: The research project showed that the drug sodium phenyl butyrate, prescribed until now for patients with alterations in the urea cycle, eases the fusion of proteins responsible for neuron connections, thus increasing the learning capacity of the mice involved. As a result, these discoveries offer new, promising perspectives for the treatment of Alzheimer's disease.

\section{Conclusion}

Alzheimer's disease, which is considered as one of the curse for mankind, is now become curable at last. Scientists make it possible by identifying the actual cause of the disease. The defective cutting pattern of Beta Amyloid Precursor Protein and dysregulation of Tau protein are mainly responsible for the disease, although mutations in different genes of chromosome-1, 14, 19, 21 are also the agents for the disease. The disease can be diagnosed by using different modern techniques like asEye test, PET scan etc. However, the Alzheimer's disease can be cured mainly by using different drugs. Among them, the most common is Memantine which is, by its action, a NMDA receptor antagonist. Other drugs like, Exelon which acts as a cholinesterase inhibitor are also useful. The drug Hydergine also reduce the harmful effects of the disease by expressing its medicinal properties like as increasing blood supply, oxygen and level of neurotransmitters in the brain cells and by its antioxidant properties. Among natural products, the leaf extract of Ginkgo biloba, is also acts as an important drug to prevent the disease by showing its antioxidant and neuroprotective effects against the plaques. Treatment with vitamins like vitamin $\mathrm{E}$ and Niacin is also proved to be useful. Hormonal treatment with Estrogen shows fruitful result. However, the peoples of Oriental countries are less affected by Alzheimer's than from peoples of Western countries. This is due to the fact that, peoples of Oriental countries take turmeric in their daily food, which prevents them from all types of Dementia. The medicinal effect of turmeric is to reduce the level by increasing macrophage activity in brain, by acting as metal chellator, by acting as antioxidant and by other important functions. Recent research have suggests that, sea drug Bryostatin, which is very useful to treat cancer, can also reduce the level of Aâ amyloid plaque formation. Some drugs like cx 516 proved to be useful in mouse model but are harmful for human body. Other researchers suggest that, the drug Sodium Phenylbuterate, which is useful in renal disorder, can help in curing Alzheimer's disease. It has also been found that, peoples who use NSAID (Non Steroidal Anti-inflammatory Drugs) are less affected by Alzheimer's disease. However, studies have shown that people, who take coffee or tea daily, are also less affected 
by the disease. A recent study with animal model indicates that caffeine appears to block several of the disruptive effects of cholesterol that make the blood-brain barrier leaky where blood-brain barrier acts as a filter to protect the brain from potentially harmful chemicals carried in the bloodstream(Chen et al.,2008).

\section{Recommendations}

Recommendations for Alzheimer disease apply to dementia disorders in general. The recommendations for the disease are clinical diagnosis, blood tests, neuroimaging, electroencephalography (EEG), cerebrospinal fluid (CSF) analysis, genetic testing, tissue biopsy, disclosure of diagnosis, treatment of Alzheimer's disease, and counseling. The specialist physician plays an important role together with primary care physicians in the multidisciplinary dementia teams, which have been established throughout Europe.

- Consume a low-fat diet.

-Eat cold-water fish (like tuna, salmon, and mackerel) rich in omega-3 fatty acids, at least 2 to 3 times per week.

-Reduce your intake of linoleic acid found in margarine, butter, and dairy products.

-Increase antioxidants like carotenoids, vitamin $\mathrm{E}$, and vitamin $\mathrm{C}$ by eating plenty of darkly colored fruits and vegetables.

- Maintain a normal blood pressure.

-Stay mentally and socially active throughout your life. -Affected persons should take drugs like Memantine, Ergoloid Mesylate, Rivastigmine Tartrate and Ginkgo biloba extracts.

-Take foods which cooked with Turmeric powder and take green tea or coffee regularly.

\section{REFERENCES}

Arnáiz, E. and Almkvist, O. (2003). Neuropsychological features of mild cognitive impairment and preclinical Alzheimer's disease. Acta Neurol. Scand., Suppl., 179: 34-41. Benke, T. (1993). Two forms of apraxia in Alzheimer's disease. Cortex, 29 (4): 715-25.

Brookmeyer, R., Johnson, E., Ziegler-Graham, K. and MH, Arrighi. (2007). Forecasting the global burden of Alzheimer's disease . Alzheimer's and Dementia, 3 (3): 186-91.

Calabrese, V., Butterfield, DA. and Stella, AM. (2003). Nutritional antioxidants and the heme oxygenase pathway of stress tolerance: novel targets for neuroprotection in Alzheimer's disease. Ital. J. Biochem., 52(4):177-81.

Chen, X., Gawryluk, J.W. Wagener, J.F., Ghribi, O. and Geiger, J.D. (2008). Caffeine blocks disruption of blood brain barrier in a rabbit model of Alzheimer's disease. The Journal of Neuroinflammation, vol.5.

Danysz, W. and Parsons, C.G. (2003).The NMDA receptor antagonist memantine as a symptomatological and neuroprotective treatment for Alzheimer's disease: preclinical evidence. International journal of Geriatric psychiatry, 18: S23-S32.

Fiala, M., Liu, P.T., Espinosa-Jeffrey, A., Rosenthal, M.J., Bernard, G., Ringman, J.M., Sayre, J., Zhang, L., Zaghi J., Dejbakhsh, S., Chiang, B., Hui, J., Mahanian, M., Baghaee, A., Hong, P. and Cashman, J. (2007). Innate immunity and transcription of MGAT-III and Toll-like receptors in Alzheimer's disease patients are improved by bisdemethoxycurcumin. Proc. Natl. Acad. Sci. US A, 104(31):12849-54.

Förstl, H. and Kurz, A. (1999). Clinical features of Alzheimer's disease. European Archives of Psychiatry and Clinical Neuroscience, 249 (6): 288-290.

Goodman, Y., Bruce, A.J., Cheng, B. and Mattson, M.P. (1996) Estrogens attenuate and corticosterone exacerbates excitotoxicity, oxidative injury, and amyloid beta-peptide toxicity in hippocampal neurons. J Neurochem, 66:1836-44.

Hernandez, F. and Avila, J. (2007). Tauopathies. Cell. Mol. Life Sci., 64 (17): 2219-33.

Kappus, H. and Diplock, A.T. (1992). Tolerance and safety of vitamin E: a toxicological position report. Free Radic BiolMed, 13:55-74.

Kemppainen, N.M., Aalto, S. and Karrasch, M., (2008). Cognitive reserve hypothesis: Pittsburgh Compound B and fluorodeoxyglucose positron emission tomography in relation to education in mild Alzheimer's disease. Ann. Neurol., 63 (1): 112-8.

Femke. M.S. Devrij, Jacquilina A. Sluijs Luisa Gregore, David F. Fischer, Wim T. J.M.C., Hermens, Dmitry, Goldgaver,Joost Verhaagen, Fred W.Van Leeuwan and Elly M. Hol. (2001) Mutant ubiquitin expressed in Alzheimer's Disease causes neuronal death. The FASEB Journal, 15:26802688.

Lott, I.T. and Head, E. (2005). Alzheimer disease and Down syndrome: factors in pathogenesis. Neurobiol. Aging, 26 (3): 383-89.

Oyama, Y., Chikahisa, L., Ueha, T., Kanemaru, K. and Noda, K. (1996). Ginkgo biloba extract protects brain neurons against oxidative stress induced by hydrogen peroxide. Brain Research, 712:349-352.

Spiegel, R. (1983). A controlled long term study with Hydergine, in healthy elderly volunteers. Journal of the American Geriatrics Society, 31: 549-555.

Tiraboschi, P., Hansen, L.A., Thal, L.J. and Corey-Bloom, J. (2004). The importance of neuritic plaques and tangles to the development and evolution of AD. Neurology, 62 (11): 1984-9. 